\title{
DISCRETIZATION INFLUENCE ON REgULARIZATION BY Two LOCALIZATION LIMITERS
}

\author{
By Antonio Huerta, ${ }^{1}$ Associate Member, ASCE, and Gilles Pijandier-Cabot, ${ }^{2}$ \\ Associate Member, ASCE
}

\begin{abstract}
AestRacr: In materials with a strain softening characteristic behavior, classical continuum mechanics favors uncontrolled strain localization in numerical analyses. Several methods have been proposed to regularize the problem. Two such localization limiters developed to overcome spurious instabilities in computational failure analysis are examined and compared. A disturbance analysis, on both models, is performed to obtain the closed form solution of propagating wave velocities as well as the velocities at which the energy travels. It also shows that in spite of forcing the same stress-strain response, the wave equation does not yield similar results. Both propagations of waves are dispersive, but the internal length of each model is different when equivalent behavior is desired. In fact, the previously suggested derivations of gradient models from nonlocal integral models were not completely rigorous. The perturbation analysis is pursued in the discrete space where computations are done, and the closed form solutions are also obtained. The finiteelement discretization introduces an added dispersion associated to the regulari zation technique. Therefore, the influence of the discretization on the localization limiters can be evaluated. The element size must be smaller than the intemal length of the models in order to obtain sufficient accuracy.
\end{abstract}

\section{INTRODUCTION}

Progressive cracking in quasi-brittle materials such as concrete often results in strain softening. Since the material loosens gradually, its stresscarrying-capability, load-versus-displacement curves exhibit a negative slope that is typical in fracture mechanics. In the context of continuum mechanics, these curves, say on a direct-tension test, are converted into stress-versusaverage-strain relations that accordingly exhibit softening, that is, a loss of positive definiteness of the material tangent operator. Since the work of Rice (1976), it is agreed that the existence of softening causes major problems due to strain localization. From a mathematical point of view, the appearance of localization in classical continuum mechanics is associated with a change in type of governing equation. For instance, in quasi-static analysis, the governing equations lose ellipticity, while in transient analyses, the dynamic equations change from hyperbolic to elliptic at the inception of localization. Thus, initial-value problems do not remain well posed. Some of the mathematical difficulties raised by this behavior are not critical, because localization is reduced to a set of measure zero, and a resolution is possible in the sense of the distributions. On the other hand, the physical and numerical difficulties are much harder to overcome. It has been pointed out often [e.g., Bažant et al. (1984)] that strain softening in classical con-

'Prof., Departamento de Matemática Aplicada III, E.T.S. de Ingenieros de Cam inos, Universitat Politècnica de Catalunya, Campus Nord C-2, E-08034 Barcelona, Spain.

${ }_{2}^{2}$ Prof., Laboratoire de Mécanique et Technologie, E.N.S. de Cachan/CNRS/Univ ersité de Paris VI and GRECO Géomatériaux, Avenue du Président Wilson, F94235 Cachan, France. 
tinuum mechanics produces failure without energy dissipation (since it occurs over a set of measure zero). This is unrealistic. In a classical continuum the strain-softening characteristic behavior favors uncontrolled strain localization in numerical analyses, and as a consequence finite-element calculations suffer from a pathological mesh-dependence.

Several regularization methods have been proposed to overcome these problems. Among these are micropolar constitutive relations (de Borst 1991), nonlocal models (Bažant et al. 1984; Pijaudier-Cabot and Bažant 1987), and gradient-dependent models (Lasry and Belytschko 1988; de Borst and Mühlhaus 1991). These constitutive relations bear the same fundamental property: an internal length, sometimes called the characteristic length, is introduced in the governing equation and scales the localization zone that cannot develop over a set of measure zero. This property was elucidated recently for gradient-plasticity models (de Borst et al. 1991) and nonlocaldamage models (Pijaudier-Cabot and Benallal 1992) using perturbation analyses, because two well-differentiated length scales are present in the problem: one associated to the macroscopic behavior of the solid and one that is microscale, related to the localization zone. There is, however, a basic underlying issue common to every constitutive model and resolution technique: the spatial interpolation of the primitive variables. Shear bands for plastic-type materials or damaged zones for brittle-type materials must be accurately described. This implies that the measure of the discretization (loosely speaking: the element size) must be of the order of the internal length, that is, the microscopic scale. This has been observed in practice on finite-element computations in statics and dynamics, where the width of the localization zone than the internal len

Sluys 1992).

The present pape the discretization error, defined as the error on the wave speeds due to fliscretization is derived as a function of the element size The method tis based on a linearized analysis of a disturbance around the equilibrium state followed by a von Neumann (or Fourier) method in the discretized domain.

Two localization limiters are investigated and compared: a nonlocal (integral) damage model and a gradient plasticity model. The constitutive relations are set in such a format that they can be compared, that is, the same stress-strain response for monotonic loading in a homogeneous strained solid is imposed. For the sake of clarifying the analytical developments, the present study is restricted to a one-dimensional continuum, although the method can be applied to a two-dimensional context without restrictions.

After comparing the constitutive relations, discretization errors in the damage model are discussed. Next the same results are examined with the gradient-plasticity model. Finally, both models are compared.

\section{CONSTITUTIVE RELATIONS}

\section{Nonlocal Damage Model}

The first limiter considered is the one-dimensional version of the nonlocalscalar-damage model proposed by Pijaudier-Cabot and Bažant (1987). The constitutive relation reads

$$
\sigma=\left(\begin{array}{ll}
1 & D
\end{array}\right) E \varepsilon
$$

in which $\sigma$ and $\varepsilon$ are the current stress and total strain, respectively; $D$ is 
the damage variable which ranges from 0 initially to 1 when the material cannot sustain any stress; and $E$ is the initial-(undamaged)- material Young's modulus. Given an equilibrium state defined by a stress field $\sigma^{0}$ with the corresponding strain $\varepsilon^{0}$ and damage $D^{0}$ fields, the rate constitutive equation is written as

$$
\dot{\sigma} \quad\left(1-D^{0}\right) E \dot{\varepsilon} \quad \dot{D} E \varepsilon^{0}
$$

where a superior dot denotes time derivatives. The growth of damage is defined as the function of the average damage energy-release rate $\bar{Y}$ and the damage loading surface, that is

$$
\begin{gathered}
f(\bar{Y})=F(\bar{Y}) \quad F\left(K^{*}\right) \\
\left\{\begin{array}{l}
\dot{D}(x)=\frac{d F(\bar{Y})}{d \bar{Y}} \dot{\bar{Y}}(x) ; \quad \text { if } f(\bar{Y})=0 \text { and } \dot{\bar{Y}}>0 \\
\dot{D}(x)=0 ; \quad \text { if } f(\bar{Y})<0 \text { or if } f(\bar{Y})=0 \text { and } \dot{\bar{Y}} \leq 0
\end{array}\right.
\end{gathered}
$$

In (3), $F$ = an experimentally determined evolution law for damage; and hardening-softening parameter that is taken as the maximum between $\bar{Y}$ at this particular point for all the previous history of loading and an initial threshold of damage. In fact, $F\left(K^{*}\right)=D$, which shows clearly the pathdependence of this nonlocal strain-softening model. Actually, this definition of growth of damage is parallel to the classical nonassociated plasticity. That is, the growth of damage can also be defined from a dissipation potential

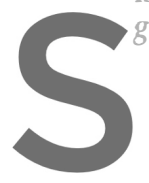

\section{, namely}
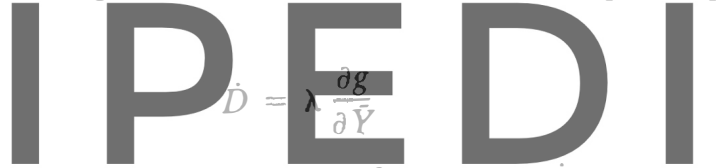

with the Kühn-Tucker conditions $\lambda \geq 0, f \leq 0$, and $\lambda \dot{f}$

0 ; and $g$

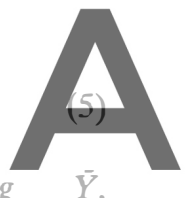

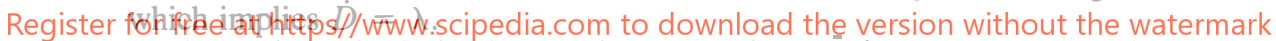

The basic variable in the previous equations $\bar{Y}$ (average energy-release rate) introduces the nonlocal nature of the model, since it is an average of the local energy-release rate $Y$ and is defined as

$$
\bar{Y}(x)=\int_{\Omega} \frac{\Psi(s-x)}{V_{r}(x)} Y(s) d s
$$

with

$$
Y(x)=\frac{1}{2} \varepsilon(x) E \varepsilon(x)
$$

where the integration domain $\Omega$ spans over the entire structure analyzed; $\Psi(s-x) / V_{r}(x)=$ a weighting function adequately defined to normalize the averaging; and $V_{r}(x)=\int_{\Omega} \Psi(s \quad x) d s$ the representative volume at point $x$. The weighting function $\Psi$ is defined as

$$
\Psi(x)=e^{-\left(|x|^{2} / 2 l_{D}^{2}\right)}
$$

where $l_{D}=$ internal length of the nonlocal continuum that can be estimated experimentally as a function of the size of the heterogeneities in the material (Bažant and Pijaudier-Cabot 1989).

With these definitions, the increase of damage from the previously cited equilibrium state around $D^{0}$ is 


$$
\dot{D}(x)=\frac{d F(\bar{Y})}{d \bar{Y}} \int_{\Omega} \frac{\Psi(s-x)}{V_{r}(x)} E \varepsilon^{0}(s) \dot{\varepsilon}(s) d s
$$

with the conditions $f(\bar{Y})=0$ and $\dot{\bar{Y}}>0$.

Once the expression for the growth of damage, (9), is known; it is possible to perform a linearized analysis of a disturbance $\dot{u}$ (velocity field) around the equilibrium state defined by the stress $\sigma^{0}$, strain $\varepsilon^{0}$, and damage $D^{0}$ fields in a strain-softening regime over a subdomain $\vartheta$ of the studied solid $\Omega$. Such an analysis has been reported in Pijaudier-Cabot and Benallal (1992) in a general three-dimensional context and here it is particularized to the present case. Two assumptions are made to solve the problem in closed form: first, the deformation, damage, and stress states are homogeneous, and second, the nonlinearity due to the growth of damage (4) is removed by imposing that the subdomain $\vartheta$ remains in the strain-softening state. Under these conditions the linearized equation of motion reads

$$
\rho \frac{\partial^{2} \dot{u}}{\partial t^{2}}=\frac{\partial \dot{\sigma}}{\partial x}
$$

\section{where $\rho=$ mass density, and the above equation is only valid in the strain- softening subdomain $\vartheta$. Possible wave solutions of (10) with a constitutive behavior defined by (2) and (9) are sought in harmonic form}

$$
\ddot{u}=A e^{-i k\left(x-c_{e} t\right)}
$$

where $A=$ amplitude of the wave; $k$ wave number; and velocity. Substitution of this valocity field into the equations yields the procedure [see Pifaudier-Cabot and Benallal (1992) derivation]. The wave speed is then given by the equation

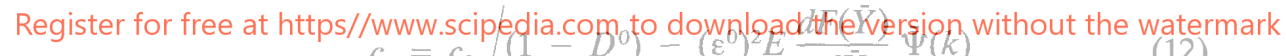
$c_{e}=c_{0}$

where $c_{0}=$ initial wave velocity in the elastic medium; $c_{0}=$ $\sqrt{E / \rho}$; and $\bar{\Psi}(k)=$ the Fourier transform of $\Psi$, which is a function of the wave number. Given the weighting function, (8), its Fourier transform reads

$$
\bar{\Psi}(k)=e^{-\left[\left(k l_{D}\right)^{2} / 2\right]}
$$

Several important conclusions can be drawn from such a result. It must be noticed that the phase velocity is dispersive; that is, waves with different wavelengths travel at different speeds. Similar results were also obtained for different localization limiters by Lasry and Belytschko (1988) and Sluys et al. (1991).

A critical value of the wave number exists for which the phase velocity vanishes, namely

$$
\bar{\Psi}(k)=\frac{1-D^{0}}{\left(\varepsilon^{0}\right)^{2} E \frac{d F(\bar{Y})}{d \bar{Y}}}
$$

These are the stationary waves obtained in statics [see Pijaudier-Cabot and Bodé (1992)]. For high frequencies, that is, wave numbers larger than this critical value, the wave velocity is real and therefore the disturbance $\dot{u}$ 
remains bounded. Asymptotically, as the wave number goes to infinity, waves with an infinite frequency propagate with the elastic wave speed of a damaged material: $\sqrt{E\left(1 \quad D^{0}\right) / \rho \text {. On the other hand, for low frequen- }}$ cies, the wave speed is imaginary and the disturbance is unbounded. These large wave lengths, however, are not able to develop in the strain-softening region of order $l_{D}$.

Finally, the critical wave number depends on the initial strain field $\varepsilon^{0}$. Before the peak stress, all the waves can propagate. After the peak stress, only short wave modes can propagate. Thus, it is expected that the damage front in a bar propagates even during softening.

Eq. (12) shows that the softening region $\vartheta$ is a dispersive medium. Hence, a difference exists between the phase velocity, that is, the velocity of a single harmonic, and the group velocity, which is the velocity at which energy travels. The group velocity, $c_{\text {group }}$, is defined by

$$
c_{\text {group }}=c_{e}+k \frac{d c_{e}}{d k}
$$

and it becomes in this particular case

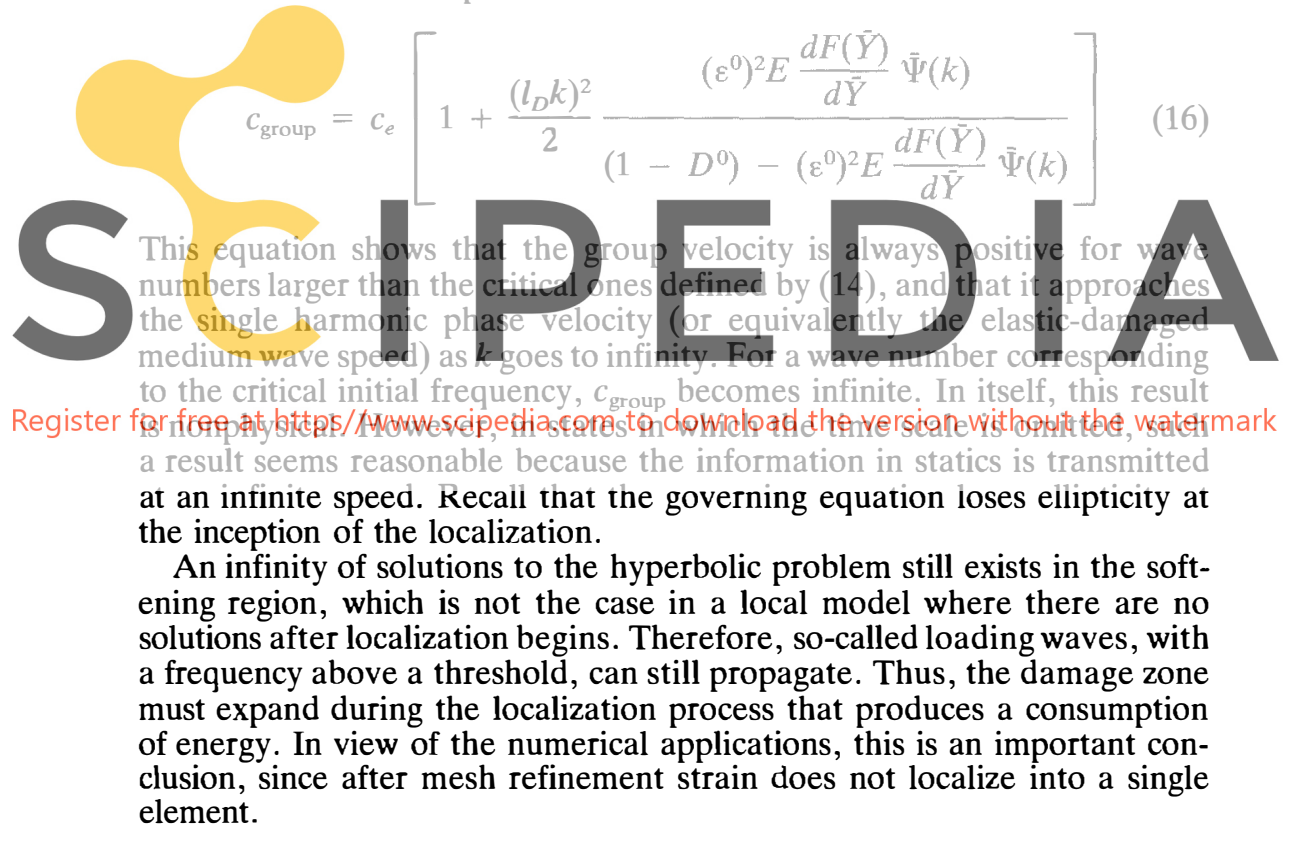

\section{Gradient Plasticity Model}

The second limiter is a nonclassical plasticity model originally proposed by de Borst and Mühlhaus (1991). The constitutive relation reads

$$
\begin{gathered}
\sigma=E \varepsilon^{e} \\
\dot{\varepsilon}=\dot{\varepsilon}^{e}+\dot{\varepsilon}^{p}
\end{gathered}
$$

with the yield function

$$
\boldsymbol{\sigma}=\sigma_{y}+H E \bar{\varepsilon}^{p}
$$


which is very similar to classical plasticity: $\sigma=$ current stress; $\sigma_{y}=$ initial yield stress; $H E=$ hardening/softening modulus, which plays a similar role for the "average" inelastic strain $\bar{\varepsilon}^{p}$ as the elastic modulus $E$ has for the elastic strain $\varepsilon^{e} . H$ is by definition negative and $|H|<1$ during softening; and it is assumed constant in the present analysis. The regularization is performed in (18) by the introduction of the variable average plastic strain $\bar{\varepsilon}^{p}$ defined from the local plastic strain $\varepsilon^{p}$ as

$$
\bar{\varepsilon}^{p}=\varepsilon^{p}+l_{G}^{2} \frac{\partial^{2} \varepsilon^{p}}{\partial x^{2}}
$$

Again, the constant $l_{G}$ is the internal length for this model. The linearized perturbation analysis similar to that performed in the previous section can be found in Sluys (1992). The rate constitutive equation for the present model may be written as

$$
\dot{\sigma}=H E\left(\dot{\varepsilon}^{p}+l_{G}^{2} \frac{\partial^{2} \varepsilon^{p}}{\partial x^{2}}\right)
$$

And the linearized equation of motion obtained after substitution of (20) into (10) is

$$
-H E l_{G}^{2}\left(\frac{\partial^{4} \dot{u}}{\partial x^{4}}-\frac{\rho}{E} \frac{\partial^{4} \dot{u}}{\partial t^{2} \partial x^{2}}\right)+\rho E(1+H) \frac{\partial^{2} \dot{u}}{\partial t^{2}}-H E \frac{\partial^{2} \dot{u}}{\partial x^{2}}=0
$$
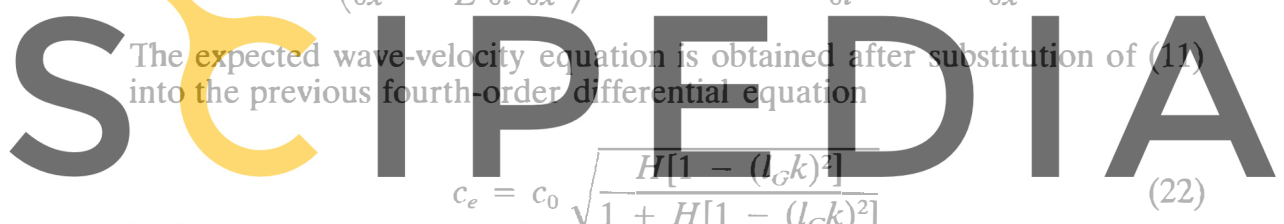

Register for free at https//www.scipedia.com to download the version without the watermark

where $c_{0}$ is again the initial wave speed in the elastic medium.

The previous equation shows that for classical plasticity (gradient-independent model, that is, $l_{G}=0$ ) the wave speed becomes imaginary as soon as softening occurs. On the other hand, for a gradient-dependent model (i.e., $l_{G} \neq 0$ ) the previous equation clearly shows the dispersion of the wave velocity. Moreover, the onset of softening does not imply an imaginary velocity. In fact, with this localization limiter, a critical value for the wave number also exists. It is defined by

$$
k=\frac{1}{l_{G}}
$$

Notice here that the critical wave number is independent of the original reference strain state from which the perturbation analysis is performed. This characteristic is clearly different from the previously presented nonlocal model.

The softening region is also for this model a dispersive medium; therefore, the group velocity must be evaluated to check for any inconsistency. Using again (15), the group velocity is written as

$$
c_{\text {group }}=c_{e}\left\{1-\frac{\left(l_{G} k\right)^{2}}{1+(1+H)\left[1-\left(l_{G} k\right)^{2}\right]}\right\}
$$


which is always positive for wave numbers larger than the critical one and becomes infinite for wave numbers equal to the critical one.

\section{Nonlocal/Gradient Relation}

Several authors (Bažant 1984; Lasry and Belytschko 1988; de Borst and Mühlhaus 1991) have suggested that gradient relations can be derived from nonlocal-integral models. They follow two basic ideas: first, the so-called average-rate inelastic strain is defined in a nonlocal manner, and second, the gradient relation is obtained after an expansion in Taylor series up to second order. As is to be shown, the last step is not completely rigorous.

First of all, $\dot{\varepsilon}^{q}$ is defined nonlocally, that is

$$
\ddot{\varepsilon}^{p}\left(x, l_{G}^{2}\right)=\int_{-\infty}^{+\infty} \frac{\Psi(s-x)}{V_{r}} \dot{\varepsilon}^{p}(s) d s
$$

with $\Psi(x)=e^{\left(|x|^{2} / 2 l_{\infty}^{2}\right)}$; and $V_{r}=\int_{-\infty}^{+x} \Psi(s-x) d s=l_{G} \sqrt{2 \pi}$, where, for the sake of simplicity, an infinite one-dimensional case is taken. With such a definition, it is easy to prove that if $\dot{\varepsilon}^{p}(x)$ is continuous and integrable, then $\lim _{l_{G \rightarrow 0}} E^{p}\left(x, l_{G}^{2}\right)=\dot{E}^{p}(x)$. Therefore, as expected, as $l_{G} \rightarrow 0$, a classical continuum is recovered and (25) represents a consistent nonlocal approach [see de Borst et al. (1991)].

Then, after a simple change of variables inside the integral of (25), namely $t=s-x$, the rate plastic strain, $\dot{\varepsilon}^{p}(x+t)$, is expanded in Taylor series from $t=0$. Since the weighting function is known, the integrals can be evaluated and the expression for the average-rate inelastic strain becomes
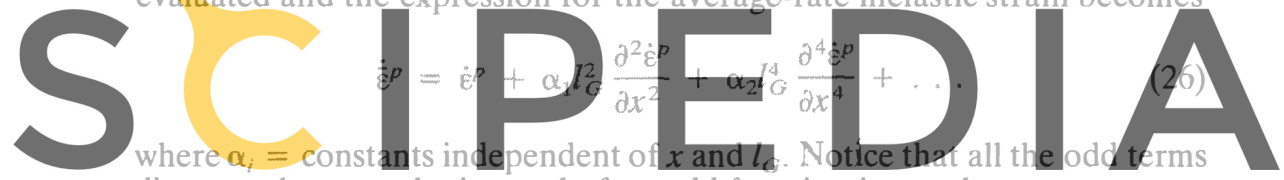

disappear because the integral of an odd function is equal to zero.

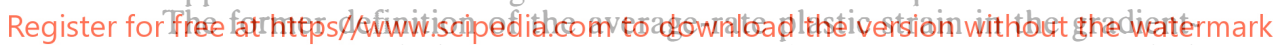
dependent model, (19), is readily obtained taking the two first terms in (26) and adjusting the definition of the weighting function by a constant such that $\alpha_{1}=1$. However, this development assumes that all the higher order terms involving even powers of $l_{G}$ and the corresponding derivatives of $\dot{\varepsilon}^{p}$ are negligible. It is based on the conjecture that $l_{G}$ is small while the derivatives are bounded. This is not vetified. In fact, the derivatives of $\dot{\varepsilon}^{p}$ grow at the same rate as $l_{G}$ decreases. In statics for instance, the derivatives of the plastic strain are in the order of, or larger than, $l_{G}{ }^{-2 i}$ for the $i$-term. Thus, they become unbounded as $l_{G} \rightarrow 0$. This result should be expected in a classical continuum theory.

According to equilibrium, the distribution of plastic strain rate is (de Borst and Mühlhaus 1991)

$$
\dot{\varepsilon}^{p}=A^{p} \cos (k x)+B^{p}
$$

where $A^{p}$ and $B^{p}$ are constants, the former associated to the amplitude of the plastic strain, and the latter derived from integration. Clearly from (27) the $i$-term in the Taylor expansion, (26), is

$$
\alpha_{i} l_{G}^{2 i} \frac{\partial^{2 i} \dot{\varepsilon}^{p}}{\partial x^{2 i}}=\alpha_{i} A^{F}(-1)^{n}\left(l_{G} k\right)^{2 i} \cos (k x)
$$

Noticing that only wave numbers larger than the critical one are allowed, that is, $k \geq 1 / l_{G}$, it is easy to show that 


$$
\alpha_{i} l_{G}^{2 i} \frac{\partial^{2 i} \dot{\varepsilon}^{p}}{\partial x^{2 i}} \geq \alpha_{i} A^{p}(-1)^{n} \cos (k x)
$$

which is not of the order of $l_{G}^{2 i}$. And even if $\alpha_{i}$ decreases, the assumption that all the higher order terms can be neglected is not rigorous.

It must be concluded from this analysis that nonlocal and gradient localization limiters are not equivalent. Therefore, the localization modes and the influence of the internal length should be different in the integral and in the gradient limiter. This is illustrated for the damage model on Fig. 1, which shows the wavelength of the localized modes in statics for the damage model. The gradient version of the nonlocal-damage model has been obtained by expanding the variation of the average energy-release rate up to second order in the same fashion as for the average plastic strain in (26)

$$
\dot{\bar{Y}}=E \varepsilon^{0} \dot{\varepsilon}+l_{D}^{2} E \varepsilon^{0} \frac{\partial^{2} \dot{\varepsilon}}{\partial x^{2}}
$$

Note that this expansion is performed in the rate form and from the homogeneous state in order to exhibit the differences between the two regularization methods. Substitution into the equation of equilibrium with the definition of stress rate, (2), and damage rate, (4), yields

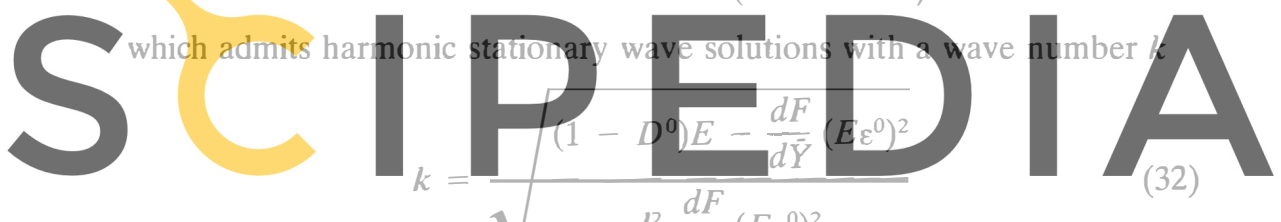

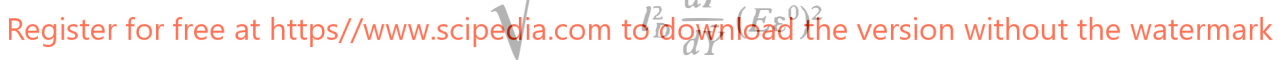

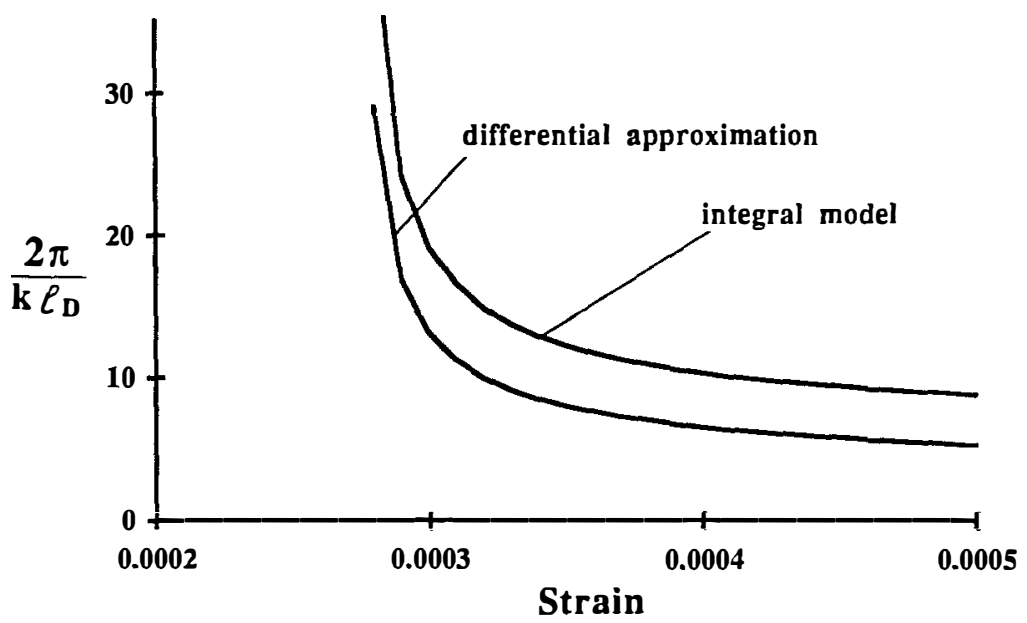

FIG. 1. Variation of Critical Wavelength with Initial State of Strain: Comparison of Integral and Differential Formulation 
In the numerical calculations, $F(\bar{Y})=1 \quad\left[1+b_{1}\left(\bar{Y}-Y_{0}\right)+b_{2}(\bar{Y}-\right.$ $\left.\left.Y_{0}\right)^{2}\right]^{1}$ with $E=32,000 \mathrm{MPa} ; \nu=0.2 ; b_{1}=605 \mathrm{MPa}{ }^{1} ; b_{2}=5.42 \times 10^{4}$ $\mathrm{MPa}{ }^{2}$, and $Y_{0}=60 \times 10^{6} \mathrm{MPa}$, which correspond to the usual values for concrete in tension. The wavelength is normalized by the inverse of characteristic length on Fig. 1. The difference between the wavelengths of the integral and differential approximation models is of the order of $30 \%$, which confirms that higher order terms in the expansion of (30) are not negligible [see also Lasry and Belytschko (1988)].

In statics, the critical wave number is the parameter that sets the width of the localization zone. If this width is assumed to be a material property, it is clear that the internal length must be a model parameter that differs from one type of localization limiter to another.

To compare quantitatively the nonlocal-damage model and the gradientplasticity model as far as discretization errors are concerned, the following methodology has been employed.

First of all, the same stress-strain-evolution law is required for both models under monotonically increasing strain. In this case, each constitutive relation reduces to a local model since $\partial^{2} \varepsilon^{P} / \partial x^{2} \quad 0$ and $\bar{Y} \quad Y$. The function $F$ in the nonlocal model, (3), is derived from this condition. That is, using (2), (3), (4), and (9), and recalling that in the present case, $D^{0} F(\bar{Y})$ and $\bar{Y}=\dot{Y}=E \varepsilon^{0} \dot{\varepsilon}$, the stress-strain relation in rate form is

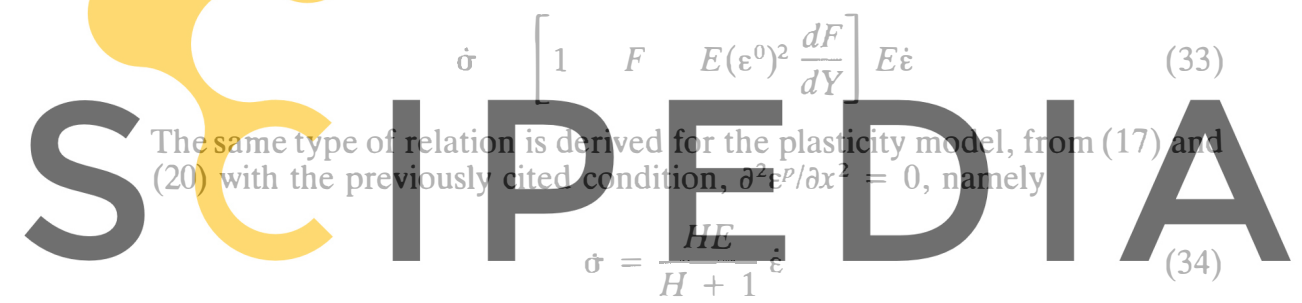

Register for free at https//www.scipedia.com to download the version without the watermark Hence, equating (33) and (34), the evolution law for damage F should satisfy the following ordinary differential equation

$$
2 Y \frac{d F}{d Y}+F-\frac{1}{1+H}=0
$$

The initial condition for this equation is defined as follows: at peak stress the material is still undamaged. The strain at peak stress is set equal to one $\left(\varepsilon^{*}=1\right)$; thus, the associated energy release rate is $\bar{Y}=E / 2$ and damage, or equivalently, $F$ must be zero. Hence, with the initial condition $F(E / 2)$ $=0$, the solution of $(35)$ is

$$
F(\bar{Y})=\frac{1}{1+H}\left(1-\sqrt{\frac{2}{E \bar{Y}}}\right)
$$

The corresponding stress-strain response, for homogeneous strain, is plotted in Fig. 2.

Second, even if this damage-evolution law induces the same stress rate for both limiters, the dispersion equation for the nonlocal damage model, (12), is still dependent on the initial strain field, while the one associated with the gradient-dependent model is independent of $\varepsilon^{0},(22)$. Another condition is then imposed. Since in statics, the first bifurcation is expected to set the width of the localization zone, the added condition is simply that 


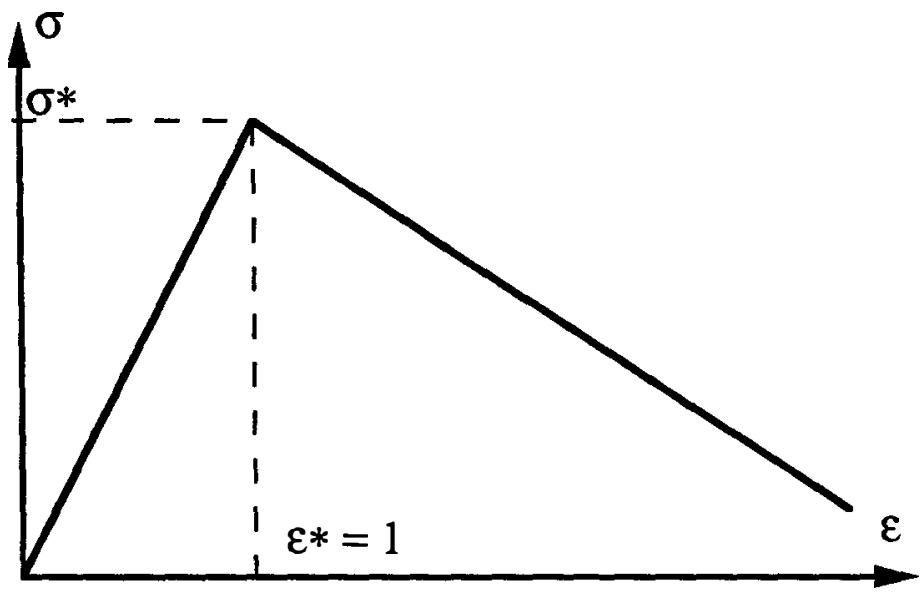

FIG. 2. Stress-Strain Curve for Two Models under Monotonically Increasing Homogeneous Strain

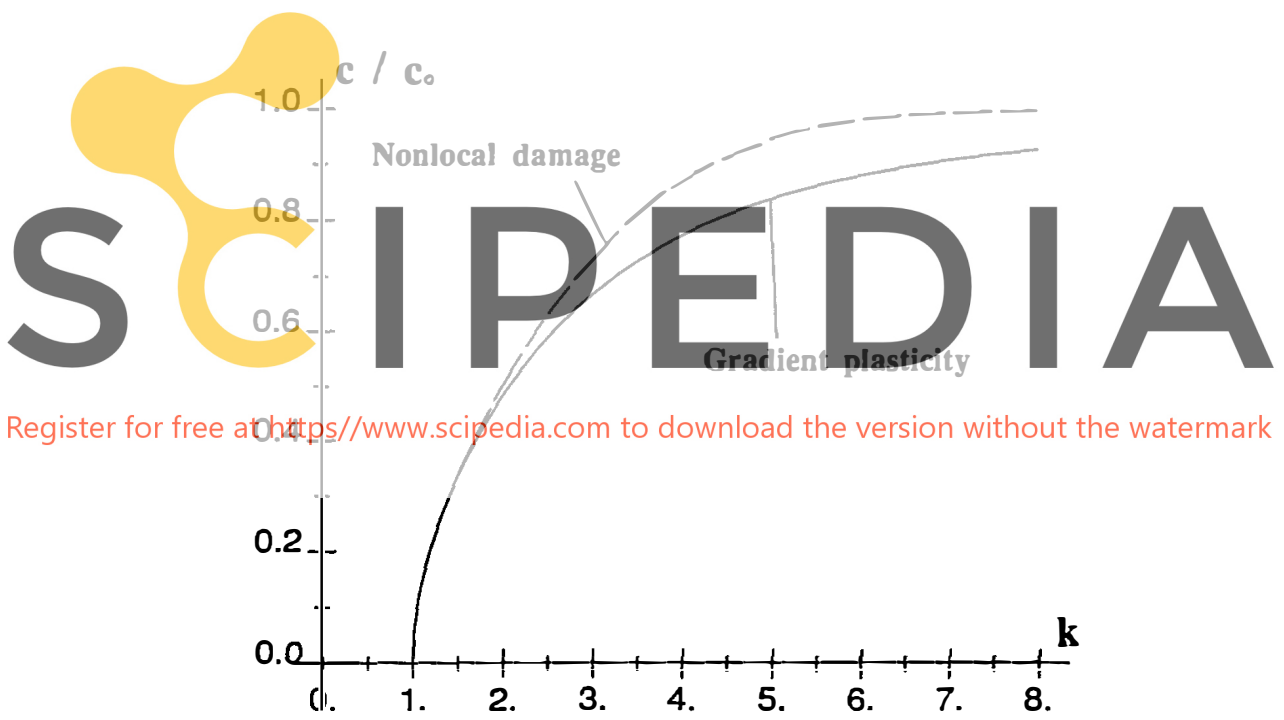

FIG. 3. Normalized Wave Velocities at $\varepsilon^{*}$ versus Wave Number

the same critical wave number [see (14) and (23)], must be obtained at this first bifurcation, namely at peak stress $\left(\varepsilon^{*}=1\right)$. A relation between the characteristic length of both models is then obtained

$$
l_{D}=l_{G} \sqrt{-2 \ln (1+H)}
$$

This result highlights again the previous remark that in order to obtain the same localization zone with two different models, even if they are relatively close, a different internal length must be used. In the following, the internal length is denoted $l_{c}$ generically for both models, although it is known for comparison purposes that the internal lengths in both models are not equal. Fig. 3 shows the wave velocities for both models normalized by the elastic wave speed, $c_{0}=\sqrt{E / \rho}$, at the peak stress as a function of the wave number. 
They present a similar behavior, although the nonlocal model reaches the elastic wave speed faster as the wave number increases.

\section{FINITE-ELEMENT REPRESENTATION: NONLOCAL MODEL}

In the previous section it was shown that propagation of loading waves is possible in the softening regime. However, this is demonstrated for a continuum domain $\Omega$ and for disturbances defined in an infinite dimensional solution space. This section deals with the same problem (linearized disturbance analysis by a von Neumann procedure) studied previously, but takes into account the finite-element representation, that is, the geometric and functional interpolation, as well as the integral-equation formulation.

To clarify the presentation, the study is again restricted to one dimension; actually, an infinite bar is analyzed. The weak form of (10) is

$$
-\int_{\vartheta} \rho \delta \dot{u} \frac{\partial^{2} \dot{u}}{\partial t^{2}} d x=\int_{\vartheta} \delta \dot{\varepsilon} \dot{\sigma} d x
$$

Notice that following the previous discussion, the analysis is restricted to the strain softening subdomain $\vartheta$. Forced boundary conditions are taken since the disturbance $\dot{u}$ is only valid in $\vartheta$. The constitutive relationship, (2) and (9), can now be introduced into (38) to deduce the complete integral equation in a classical displacement formulation

$$
\begin{aligned}
&-\int_{\vartheta} \rho \delta \dot{u} \frac{\partial^{2} \dot{u}}{\partial t^{2}} d x=\int_{\vartheta} \delta \dot{u}\left(1-D^{0}\right) E \dot{\varepsilon}(x)-E \varepsilon^{0} \frac{d F(\bar{Y})}{d \bar{Y}} \\
&\left.\cdot \int_{\Omega} \frac{\Psi(s \quad x)}{V_{r}(x)} E \varepsilon^{0} \dot{\varepsilon}(s) d s\right] d x
\end{aligned}
$$

At this point, a particular finite-element representation is chosen. It consists of constant length elements of size $h$ with linear interpolation functions. The integration of the mass matrix can be readily done. For the integration and special assembly of the discrete-stiffness matrix see Pijaudier-Cabot and Huerta (1991). After some algebraic deductions, the discrete equation of motion at node $j$ is obtained

$$
\begin{aligned}
\rho h \frac{\partial^{2} \dot{U}_{j}}{\partial t^{2}}=\frac{\left(1-D^{0}\right) E}{h}\left(\dot{U}_{j} 1-2 \dot{U}_{j}+2 \dot{U}_{j+1}\right) \\
+\left(E \varepsilon^{0}\right)^{2} \frac{d F(\bar{Y})}{d \bar{Y}}\left[\sum_{m=-\infty}^{+\infty} \beta_{j m}\left(\dot{U}_{m} 1-2 \dot{U}_{m}+\dot{U}_{m+1}\right)\right]
\end{aligned}
$$

where $\dot{U}_{j}=$ interpolation of the disturbance at node $j$, that is, $\dot{U}_{j}=\dot{u}\left(x_{j}\right)$; the parameter $\beta_{j m}$ is simply $\beta_{j m}=h \Psi\left(x_{j} \quad x_{m}\right) / V_{r}\left(x_{j}\right)$. The nonlocal term, the last integral in (39), has been integrated, as the others, by a one-point quadrature rule.

Finally, the linearly interpolated form of the exact disturbance field defined by (11) is substituted into (40), and the discrete von Neumann analysis can be performed. Notice that the aim is not to evaluate the accuracy of the time-integration technique, which is assumed to remain exact, but to study the influence of the spatial discretization. After some algebra, the 
phase velocity of the disturbance is derived taking into account the finiteelement representation

$$
c=c_{0} \frac{\sqrt{2[1 \cos (k h)]}}{k h} \sqrt{\left(1 \quad D^{0}\right)-\left(\varepsilon^{0}\right)^{2} E \frac{d F(\bar{Y})}{d \bar{Y}} h \sum_{m=-\infty}^{+\infty} \beta_{j m} e^{-i k(j-m) h}}
$$

This equation shows clearly, and as expected, that discretization introduces numerical dispersion as a function of $k h$. This dispersion is geometrically added to the one introduced by the regularization. Moreover, the integral term of the nonlocal model requires numerical integration, and the effects of such an approximation on the wave velocity are also included in (41). The summation in this equation is simply the discrete Fourier transform of the averaging function. If this Fourier transform is computed exactly, the phase velocity results in

$$
c=c_{0} \frac{\sqrt{2[1 \cos (k h)]}}{k h} \sqrt{\left(1-D^{0}\right)-\left(\varepsilon^{0}\right)^{2} E-\frac{d F(\bar{Y})}{d \bar{Y}} \bar{\Psi}(k)}
$$

which is the exact phase velocity modified by the classical dispersion term obtained for a discrete elastic medium.

Fig. 4 shows the evolution of the phase velocity in the discrete model normalized by the exact velocity denoted by $c_{e}$ for wave number $k=1.5$, and an initial strain $\varepsilon^{0}=1=\varepsilon^{*}$ (strain field corresponding to the peak stress). This figure shows that the finite-element discretization induces the expected dispersion and large errors compared with the exact velocity. When an exact integration of the nonlocal term is performed, the ratio $c / c_{e}$ does not present a truncation even if it departs from 1 very rapidly. The discrete Fourier transform induces the usual truncation and therefore larger errors. Actually, some aliasing may be expected, although the size of the localization prevents its influence in the numerical computations. Accurate results can only be expected when the size of the elements is smaller than the internal

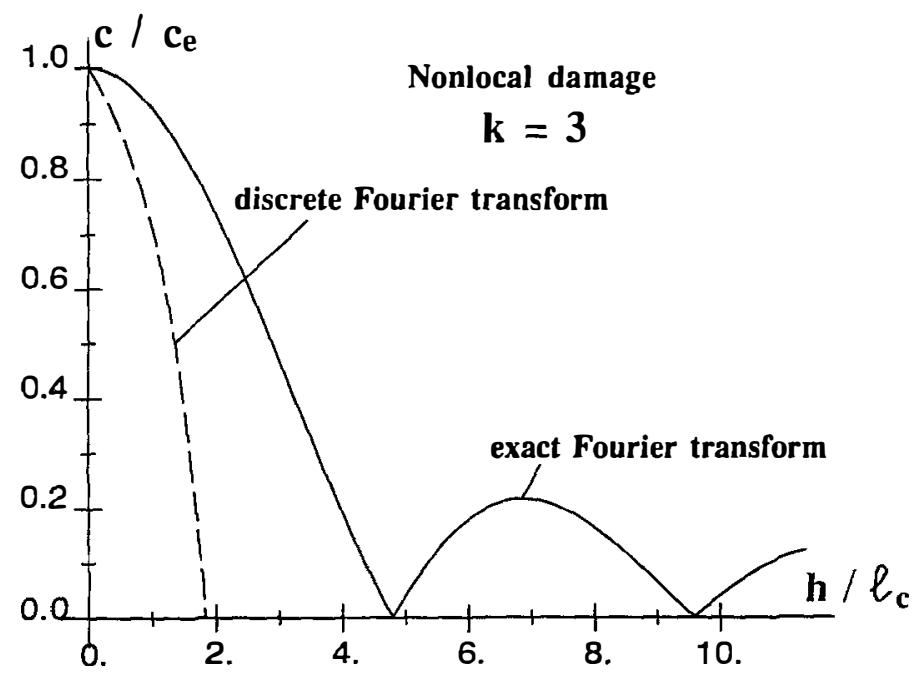

FIG. 4. Damage Model: Discretization Influence at $\varepsilon^{0}=\varepsilon^{*}$ 
length of the model $l_{c}$; thus, in the microscale order, which is much smaller than the real structure size.

Fig. 5 shows the evolution of the phase velocities as the wave number and the initial strain state $\varepsilon^{0}$ increase. Although Fig. 5(a) shows that the error is constant for different wave numbers, (41) demonstrates the classical results, specifically, that the larger the wave number, the larger the expected error. This is exemplified in Fig. 5(b), where calculations have been performed for $\varepsilon^{0}=6 \varepsilon^{*}$. When $\varepsilon^{0}$ is in the order of $\varepsilon^{*}$, the error introduced by the discretization seems to be partly compensated by the error in the discrete Fourier transform when $k$ is growing. In finite-element computa-
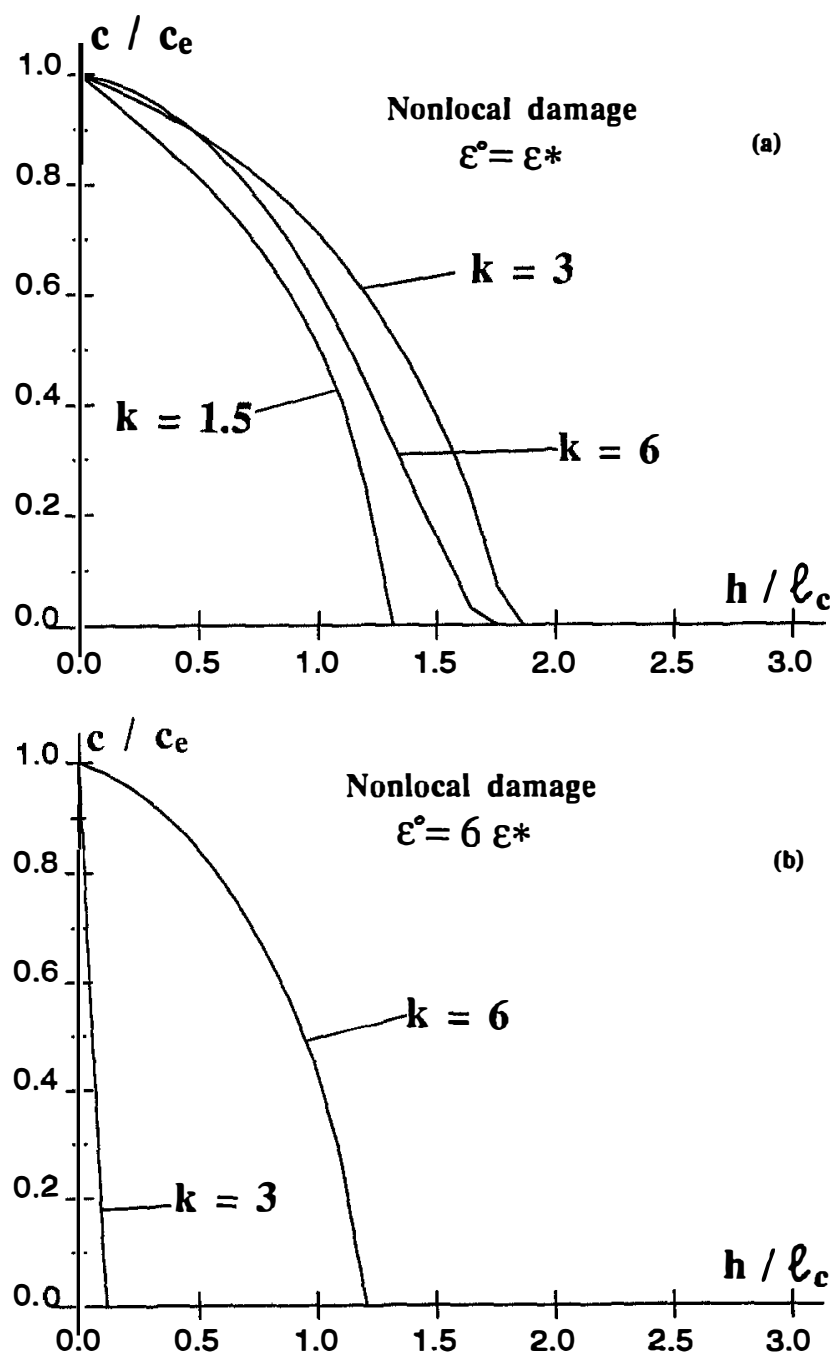

FIG. 5. Damage Model: Comparisons of Discretization Influence for Different Wave Numbers: (a) $\varepsilon^{0}=\varepsilon^{\star} ;(b) \varepsilon^{0}=6 \varepsilon^{*}$ 
tions, this presents no practical use, since damage is expected to grow rapidly along with the strain $\varepsilon^{0}$.

\section{FINITE-ELEMENT REPRESENTATION: GRADIENT-DEPENDENT MODEL}

Due to the fact that second-order spatial derivatives of the inelastic strain field are needed in this model, the finite-element description differs from the previous example. The weak form of the equation of motion is standard

$$
-\int_{\vartheta} \rho \delta \dot{u} \frac{\partial^{2} \dot{u}}{\partial t^{2}} d x=\int_{\vartheta} \delta \dot{\varepsilon} E\left(\dot{\varepsilon}-\varepsilon^{p}\right) d x
$$

The constitutive relations, and more specifically the yield condition, (18), is not local in space, but it is a second-order differential equation. A numerical scheme proposed by de Borst and Mühlhaus (1992) relaxes the consistency condition at yielding and satisfies it through a weighted residual, that is, weak form

$$
\int_{\vartheta} \delta \dot{\varepsilon}^{p}\left[E\left(\dot{\varepsilon}-\dot{\varepsilon}^{p}\right)-H E \dot{\varepsilon}^{p}-H E l_{G}^{2} \frac{\partial^{2} \dot{\varepsilon}^{p}}{\partial x^{2}}\right] d x=0
$$

where the trial functions are arbitrarily chosen in the same space of the solution $\dot{\varepsilon}^{p}$. The presence of second-order derivatives of $\dot{\varepsilon}^{p}$ in (44) requires continuous derivatives of the inelastic strain; thus, a Hermite interpolation for $\dot{\varepsilon}^{p}$ is taken. That is, for an element going from node $j$ to $j+1$ the perturbation of the inelastic strain is interpolated by

$$
\dot{\boldsymbol{\varepsilon}}^{p}(x)=\left[\bar{H}_{1}(x) \bar{H}_{2}(x) \bar{H}_{3}(x) \bar{H}_{4}(x)\right] \cdot\left(\begin{array}{c}
\dot{\Gamma}^{p} \\
\dot{\Gamma}_{j, x}^{p} \\
\dot{\Gamma}_{j+1}^{p} \\
\dot{\Gamma}_{j+1, x}^{p+1}
\end{array}\right)
$$

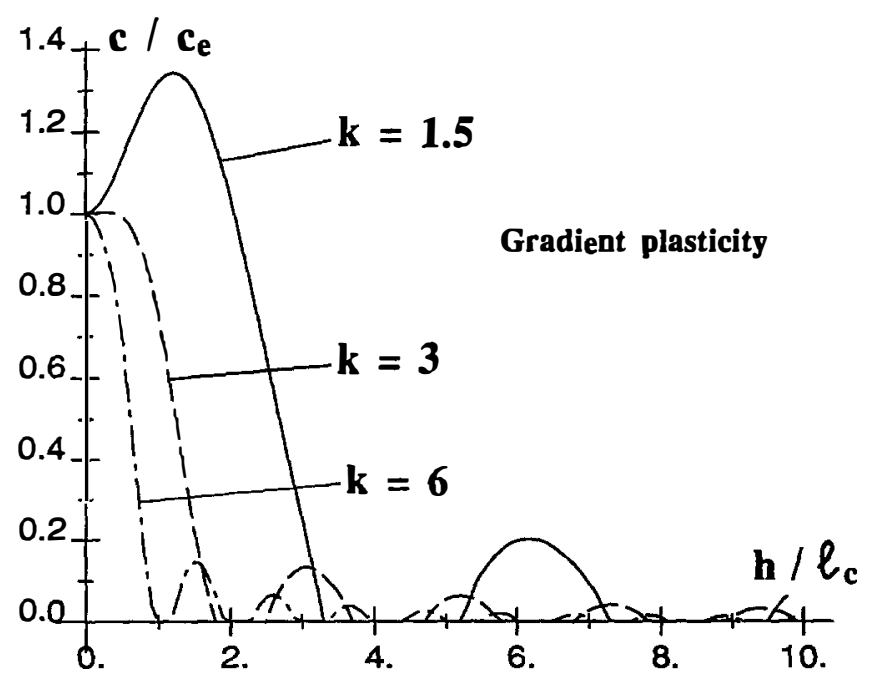

FIG. 6. Gradient-Plasticity Model: Discretization Influence on Phase Velocity 
where $\dot{\Gamma}_{j}^{p}$ and $\dot{\Gamma}_{j, x}^{p}=$ nodal values at node $j$ of the perturbation of the inelastic strain and its gradient, respectively. The interpolation functions can be found in Appendix I. The discrete equations for this model have been derived by de Borst and Mühlhaus (1992) using a linear interpolation for the nodal displacements. In the present disturbance analysis, where boundary conditions are purposely omitted since they do not modify the final result, the governing discrete ordinary differential equations reduce to

$$
\left(\begin{array}{cc}
\mathbf{M} & \mathbf{0} \\
\mathbf{0} & \mathbf{0}
\end{array}\right)\left\{\begin{array}{c}
\frac{\partial^{2} \dot{U}}{\partial t^{2}} \\
\frac{\partial^{2} \dot{\Gamma}^{p}}{\partial t^{2}}
\end{array}\right\}+\left(\begin{array}{cc}
\mathbf{K} & \mathbf{K}^{p} \\
\mathbf{K}^{p T} & \mathbf{K}^{p p}
\end{array}\right)\left\{\begin{array}{c}
\dot{U} \\
\dot{\Gamma}^{p}
\end{array}\right\}=\mathbf{0}
$$

where $\mathbf{M}=$ consistent mass matrix $\dot{\mathbf{U}}=$ vector containing the interpolation of the disturbance at each node; $\dot{\boldsymbol{\Gamma}}^{p}=$ vector containing the nodal values of the plastic strain and the first spatial derivative.

For each element, the matrix $\mathbf{K}$ is $2 \times 2, \mathbf{K}^{p}$ is $2 \times 4$, and $\mathbf{K}^{p p}$ is $4 \times 4$. In the particular case of constant-length elements, these matrices are identical for each element. Hence, after exact integration and matrix assembly, three linear algebra equations are obtained at each node

$$
\begin{aligned}
& \rho h \frac{\partial^{2} \dot{U}_{j}}{\partial t^{2}}=\frac{E}{h}\left(\dot{U}_{j-1}-2 \dot{U}_{j}+\dot{U}_{j+1}+\frac{h}{2} \dot{\Gamma}_{j-1}^{p}\right. \\
& \left.+\frac{h^{2}}{12} \dot{\Gamma}_{j-1, x}^{p}-\frac{h^{2}}{6} \dot{\Gamma}_{j, x}^{p} \frac{h}{2} \dot{\Gamma}_{j+1}^{p}+\frac{h^{2}}{12} \dot{\Gamma}_{j+1, x}^{p}\right) \\
& K_{13}^{p} \dot{U}_{j 1}+\left(K_{11}^{p}+K_{23}^{p}\right) \dot{U}_{j}+K_{21}^{p} \dot{U}_{j+1}=K_{31}^{p p} \dot{\Gamma}_{j-1}^{p}+K_{32}^{p p} \dot{\Gamma}_{j-1, x}^{p} \\
& \quad+\left(K_{11}^{p p}+K_{33}^{p p}\right) \dot{\Gamma}_{j}^{p}+\left(K_{34}^{p p}+K_{12}^{p p}\right) \dot{\Gamma}_{j, x}^{p}+K_{13}^{p p} \dot{\Gamma}_{j+1}^{p p}+K_{14}^{p p} \dot{\Gamma}_{j+1, x}^{p} \\
& K_{14}^{p} \dot{U}_{j 1}+\left(K_{24}^{p}+K_{12}^{p}\right) \dot{U}_{j}+K_{22}^{p} \dot{U}_{j+1}=K_{41}^{p p} \dot{\Gamma}_{j-1}^{p}+K_{42}^{p p} \dot{\Gamma}_{j-1, x}^{p} \\
& \quad+\left(K_{43}^{p p}+K_{21}^{p p}\right) \dot{\Gamma}_{j}^{p}+\left(K_{44}^{p p}+K_{22}^{p p}\right) \dot{\Gamma}_{j, x}^{p}+K_{23}^{p p} \dot{\Gamma}_{j+1}^{p}+K_{24}^{p p} \dot{\Gamma}_{j+1, x}^{p}
\end{aligned}
$$

At this point, the exact disturbance solution needs to be replaced in (47). Simple considerations show that the solution must be of the form

$$
\begin{aligned}
& \left.\dot{U}_{j}=A e^{i k\left(x_{j}\right.} c t\right) \\
& \left.\dot{\Gamma}_{j}^{p}=i k \Lambda_{1} A e^{-i k\left(x_{j}\right.} \text { ct }\right) \\
& \left.\dot{\Gamma}_{j, x}^{p}=-k^{2} \Lambda_{2} A e^{-i k\left(x_{j}\right.} \quad c t\right)
\end{aligned}
$$

where $\Lambda_{1}$ and $\Lambda_{2}$ are unknown constants. Physically, $\Lambda_{1}$ should represent the portion of the plastic strain over the total strain. After some algebra, the phase velocity of the disturbance in the softening region can be computed from

$$
c=c_{0} \sqrt{\frac{1}{(k h)^{2}}\left\{2[1-\cos (k h)]\left[1+\frac{\Lambda_{2}}{12}(k h)^{2}\right]-\Lambda_{1} k h \sin (k h)\right\}}
$$

which is deduced directly from the discrete form of the equilibrium equation, (47a). The two parameters $\Lambda_{1}$ and $\Lambda_{2}$ are deduced directly from the two 


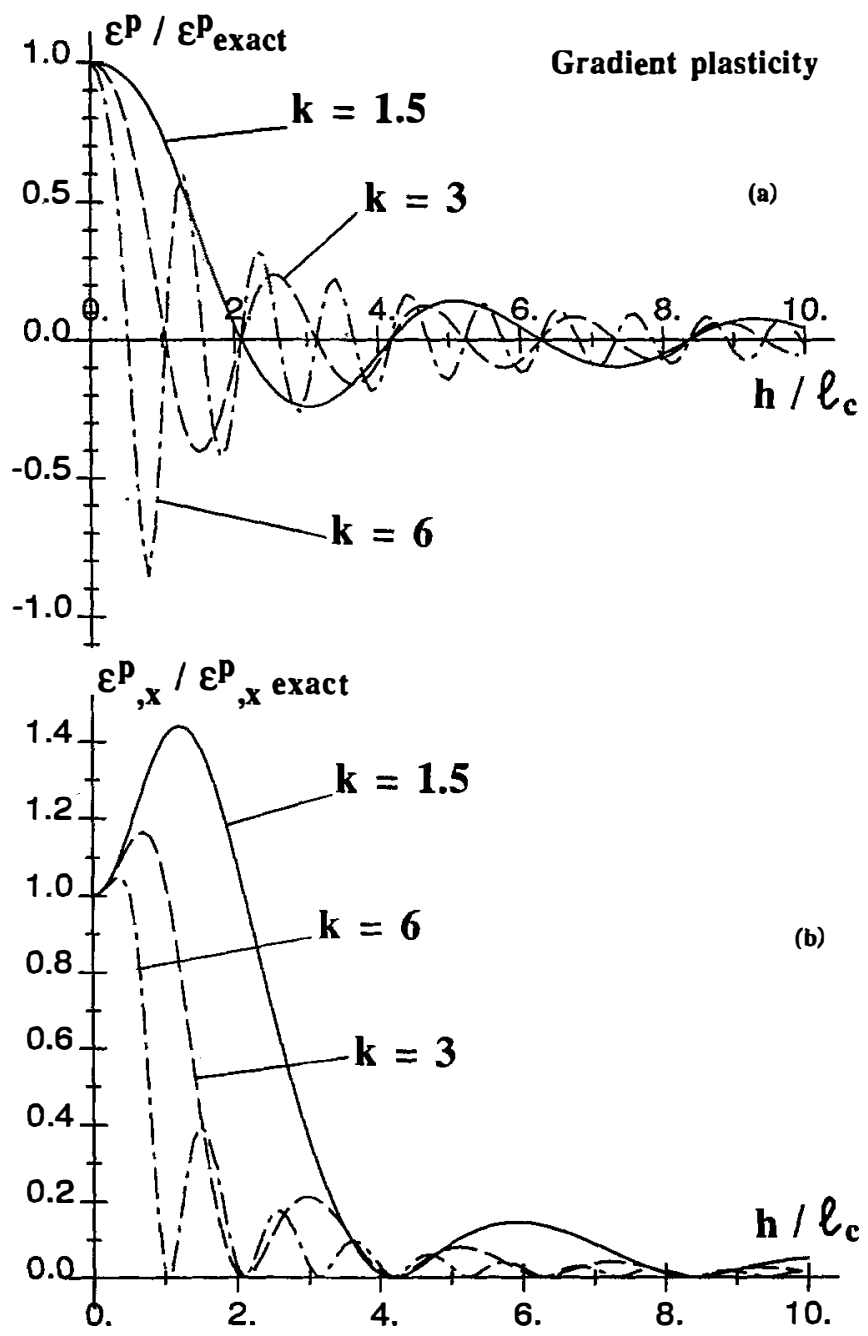

FIG. 7. Gradient-Plasticity Model: (a) Discretization Influence on Plastic Strain; (b) Discretization Influence on Plastic-Strain Gradient

discrete equations $(47 b)$ and $(47 c)$, one associated to the inelastic strain and one associated to the gradient of inelastic strain. They read

$$
\begin{aligned}
\frac{h}{6}[1-\cos (k h)]= & 2 \Lambda_{1} k \sin (k h)\left[\frac{13}{420} h^{2}(1+H)+\frac{k H l_{G}^{2}}{2}\right] \\
& +2 \Lambda_{2} k^{2}\left\{\frac{h^{3}}{105}(1+H) \quad \frac{2}{2} h H l_{G}^{2}\right. \\
& \left.-\left[1-\frac{(k h)^{2}}{2}\right]\left[\frac{h^{3}}{140}(1+H)-\frac{1}{30} H l_{G}^{2}\right]\right\}
\end{aligned}
$$




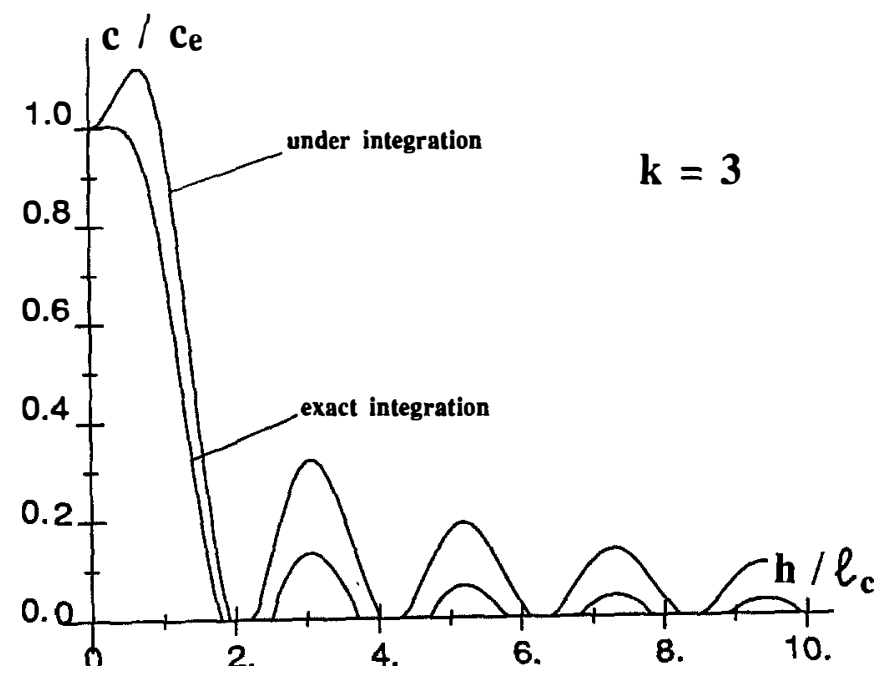

FIG. 8. Gradient-Plasticity Model: Effect of Under-Integration

$$
\begin{aligned}
\sin (k h) & 2 \Lambda_{1} k\left\{\left[\frac{9}{70} h(1+H)+\frac{6}{5 h} H l_{G}^{2}\right] \cos (k h)+\left[\frac{13}{35} h(1+H)\right.\right. \\
& \left.\left.-\frac{6}{5 h} H l_{G}^{2}\right]\right\}+2 \Lambda_{2} k^{2}\left[\frac{13}{210} h^{2}(1+H)+\frac{2}{10} l_{G}^{2}\right] \sin (k h)
\end{aligned}
$$

The limiting analysis, $h$ going to zero and $k$ finite, may be done to check the previous equations and verify the so-called exact values of both parameters $\Lambda_{1}$ and $\Lambda_{2}$; they are

$$
\Lambda_{\text {1exact }}=\frac{1}{1+H\left[1-\left(l_{G} k\right)^{2}\right]} ; \quad \Lambda_{\text {2exact }}=\Lambda_{\text {lexact }}
$$

The ratio between this "finite-element" wave velocity, (49), and the exact one $c_{e}$ is plotted in Fig. 6 for several wave numbers. The softening parameters is chosen such that $H \quad 0.1$. The element size must be smaller than the internal length of the model if accurate results are desired. The wave number has a strong influence on the wave speed, too. For low wave numbers, the phase velocity can be overestimated. Convergence toward the exact solution is not monotonic. This is not surprising, since the weak formulation of the yield condition does not guaranty monotonic convergence.

These conclusions can be extended to the evaluation of the plastic-strain rate and its gradient as shown in Fig. 7. The finite-element discretization exhibits an even larger error as the sign of the plastic strain can be opposite of the exact one (continuous model). This numerical approximation suggests that plastic strains may simply decrease while the yield condition is satisfied.

Obviously, the present results are dependent on the interpolation function and the intergration schemes. It can be noticed that matrix $\mathbf{K}^{p p}$ is not symmetric. However, (40) remains identical when the symmetric part of $\mathbf{K}^{p P}$ is employed instead of the original unsymmetrical matrix. It can be further suggested that the interpolation orders are not consistent, since a cubic polynomial is used for the plastic part of the disturbance, and a linear 

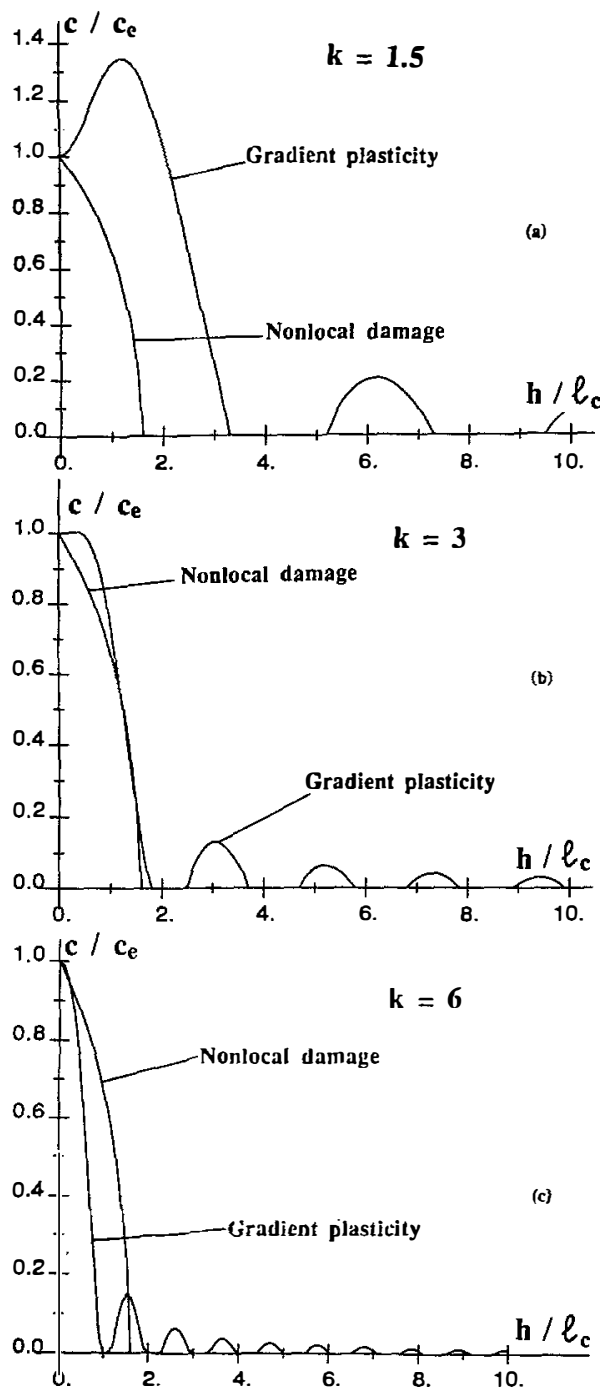

FIG. 9. Comparison of Discretization Influence at $\varepsilon^{\star}$ on Phase Velocity with Both Models for Wave Numbers Equal to (a) 1.5; (b) 3; (c) 6

interpolation is performed for the disturbance itself. Under-integration of the plastic strain may alleviate this inconsistency in the momentum equation, (43). Fig. 8 shows the error on the phase velocity when the plastic strain rate $\dot{\varepsilon}^{p}$ is under-integrated by a one point quadrature rule. Comparison with the exact integration scheme demonstrates that the error is even larger.

Finally, Fig. 9 compares the phase velocities obtained for both models at three different wave numbers. The initial strain state is at the peak stress $\varepsilon^{0}=\varepsilon^{*}$.

Although slight differences exist, namely that the discretization of the gradient-dependent model captures waves with low wave numbers more accurately, both models require an element size that is smaller than their 
internal length. On the other hand, the plasticity model can overestimate the wave velocity, which is not the case in the integral model.

\section{CONCLUSIONS}

Two types of localization limiter have been compared. Although they seem to be relatively close, with the stress-strain response under monotonic loading and a constant homogeneous strain field the same, both models yield different dispersion equations. Moreover, for the damage model, the phase velocity is a function of the state of strain, and this is not the case in the plasticity model.

Both constitutive relations contain an internal length scale that defines the width of the localization zone. However, given the width of the shear band, different internal lengths are derived for each model. This underscores the fact that the internal length must be viewed as a model parameter. In fact, the differences between the internal length parameters are associated with the manner in which the nonlocal information is introduced in the model, that is, averaging in the damage model and using a second-order gradient in the plasticity model. In fact, the previously suggested derivations of gradient models from nonlocal-integral models were not completely rigorous. The localization modes and the influence of the internal length should be different in each limiter.

The finite-element discretization produces dispersion. With the nonlocal model, this dispersion can be divided into two parts: one is classical and it is associated to the usual discretization of elliptic operators; and the other is due to the nonlocal term and it is associated to a discrete Fourier transform.

The finite-element discretization with the gradient-plasticity model yields also a dispersion equation. Convergence toward the exact solution as the element size tends to zero is not monotonic. Large errors are observed in the plastic strain rate.

The present study shows that, for both models, the size of the finite elements must be smaller than the internal length in order to achieve good accuracy. In transient finite-element analysis, which focuses on capturing accurately the propagation of the damage or plastic strain fronts, these results suggest that finite elements should be very small compared to the size of the structure. This underlines clearly the need for adaptive remeshing strategies at least in the localization area where the anelastic behavior may damp out any spurious reflections of short enough wavelengths [see Bažant (1978)].

\section{ACKNOWLEDGMENTS}

Partial financial support from the France-Spain Integrated Action program and from the EURO-GRECO "Geomaterials" under the Science program of the Commission of the European Communities are gratefully acknowledged.

\section{APPENDIX I. HERMITIAN INTERPOLATION OF THE PLASTIC STRAIN}

Consider a finite element of length $h$ going from node $j$ to node $j+1$. The plasticstrain disturbance is interpolated with the following polynomials: 


$$
\dot{\varepsilon}^{p}(x)=\left[\bar{H}_{1}(x) \bar{H}_{2}(x) \bar{H}_{3}(x) \bar{H}_{4}(x)\right] \cdot\left(\begin{array}{c}
\dot{\Gamma}_{j}^{p} \\
\dot{\Gamma}_{j, x}^{p} \\
\dot{\Gamma}_{j+1}^{p} \\
\dot{\Gamma}_{j+1, x}^{p}
\end{array}\right)
$$

with

$$
\begin{aligned}
& \bar{H}_{1}=1 \quad 3\left(\frac{x-x_{j}}{h}\right)^{2}+2\left(\frac{x-x_{j}}{h}\right)^{3} \\
& \bar{H}_{2}=\left[\left(\frac{x-x_{j}}{h}\right)-2\left(\frac{x-x_{j}}{h}\right)^{2}+\left(\frac{x-x_{j}}{h}\right)^{3}\right] h \\
& \bar{H}_{3}=3\left(\frac{x-x_{j}}{h}\right)^{2}-2\left(\frac{x-x_{j}}{h}\right)^{3} \\
& \tilde{H}_{4}=\left[-\left(\frac{x-x_{j}}{h}\right)^{2}+\left(\frac{x-x_{j}}{h}\right)^{3}\right] h
\end{aligned}
$$

in the global coordinate system where the initial coordinate of the first node is $x_{j}, h=x_{j+1} \quad x_{j}$ and $x_{j} \leq x \leq x_{j+1}$.

\section{APPENDIX II. REFERENCES}

Bažant, Z. P. (1978). "Spurious reflection of elastic waves in nonuniform finite element grids." Comp. Methods in Appl. Mech. and Engrg., 13, 91-100.

Bažant, Z. P. (1984). "Imbricate continuum and its variational derivation." J. Engrg. Mech., ASCE, 110(12), 16931712.

Bažant, Z. P., Belytschko, T., and Chang, T. P. (1984). "Continuum theory for strain softening." J. Engrg. Mech., ASCE, 110(12), 1666-1692.

Bažant, Z. P., and Pijaudier-Cabot, G. (1989). "Measurement of characteristic length of nonlocal continuum." J. Engrg. Mech., ASCE, 115(4), 755767.

de Borst, R. (1991). "Simulation of strain localization: a reappraisal of the Cosserat continuum." Engrg. Computations, 8, 317-332.

de Borst, R., Huerta, A., and Pijaudier-Cabot, G. (1991). "Localization limiters: properties implementation and solution control." Rep. No. 25-2-91-209, Delft Univ. of Technology, Delft, The Netherlands.

de Borst, R., and Mühlhaus, H. B. (1991). "Continuum models for discontinuous media." Fracture Processes in Concrete, Rock and Ceramics, Vol. 2, J. G. M. Van Mier et al., eds., 601-618.

de Borst, R., and Mühlhaus, H. B. (1992) "Gradient dependent plasticity: formulation and algorithmics aspects." Int. J. Numerical Methods in Engrg., 35, 521 539.

Lasry, D., and Belytschko, T. (1988). "Localization limiters in transient problems." Int. J. Solids and Struct., 24(6), 581597.

Pijaudier-Cabot, G., and Bažant, Z. P. (1987). "Nonlocal damage theory." J. Engrg. Mech., ASCE, 118(10), 15121533.

Pijaudier-Cabot, G., and Benallal, A. (1992). "Strain localization and bifurcation in a nonlocal continuum.” Internal Rep., Laboratoire de Mécanique et Técnologie, Cachan, France.

Pijaudier-Cabot, G., and Bodé, L. (1992). "Localization in a nonlocal continuum." Mech. Res. Communications, 19(2), 145153.

Pijaudier-Cabot, G., and Huerta, A. (1991). "Finite element analysis of bifurcation 
in nonlocal strain softening solids." Comp. Methods in Appl. Mech. and Engrg., 90, 905-919.

Rice, J. R. (1976). "The localization of deformation." Theoretical and Applied Me chanics. W. T. Koiter, ed., North Holland Publishing Co., Amsterdam, The Netherlands.

Sluys, L. J. (1992). "Wave Propagation, Localization and Dispersion in Softening Solids," PhD thesis, Delft University of Technology, Delft, The Netherlands.

Sluys, L. J., de Borst, R., and Mühlhaus, H. B. (1991). "Wave propagation and localization in a gradient-dependent elastic-plastic solid." Int. Conf. on Nonlinear Engrg. Computations, Split, Croatia. 